\title{
LEFSCHETZ THEORY FOR EXTERIOR ALGEBRAS AND FERMIONIC DIAGONAL COINVARIANTS
}

\author{
JONGWON KIM AND BRENDON RHOADES
}

\begin{abstract}
Let $W$ be an irreducible complex reflection group acting on its reflection representation $V$. We consider the doubly graded action of $W$ on the exterior algebra $\wedge\left(V \oplus V^{*}\right)$ as well as its quotient $D R_{W}:=\wedge\left(V \oplus V^{*}\right) /\left\langle\wedge\left(V \oplus V^{*}\right)_{+}^{W}\right\rangle$ by the ideal generated by its homogeneous $W$ invariants with vanishing constant term. We describe the bigraded isomorphism type of $D R_{W}$; when $W=\mathfrak{S}_{n}$ is the symmetric group, the answer is a difference of Kronecker products of hookshaped $\mathfrak{S}_{n}$-modules. We relate the Hilbert series of $D R_{W}$ to the (type A) Catalan and Narayana numbers and describe a standard monomial basis of $D R_{W}$ using a variant of Motzkin paths. Our methods are type-uniform and involve a Lefschetz-like theory which applies to the exterior algebra $\wedge\left(V \oplus V^{*}\right)$.
\end{abstract}

\section{INTRODUCTION}

Let $\mathbb{C}\left[x_{1}, \ldots, x_{n}, y_{1}, \ldots, y_{n}\right]$ be a polynomial ring in $2 n$ variables equipped with the diagonal action of the symmetric group $\mathfrak{S}_{n}$ :

$$
w \cdot x_{i}:=x_{w(i)} \quad \text { and } \quad w \cdot y_{i}:=y_{w(i)}
$$

for all $w \in \mathfrak{S}_{n}$ and $1 \leq i \leq n$. The quotient of $\mathbb{C}\left[x_{1}, \ldots, x_{n}, y_{1}, \ldots, y_{n}\right]$ by the ideal generated by the homogeneous $\mathfrak{S}_{n}$-invariants of positive degree is the diagonal coinvariant ring; its bigraded $\mathfrak{S}_{n}$-structure was calculated by Haiman [10] using algebraic geometry.

In the last couple years, algebraic combinatorialists have studied variations of the diagonal coinvariants involving sets of commuting and anti-commuting variables [3, 5, 8, 14, 16, 18, 19. In this paper we completely describe the bigraded $\mathfrak{S}_{n}$-structure of the diagonal coinvariants involving two sets of anti-commuting variables (but no commuting variables). Our methods apply equally well (and uniformly) to any irreducible complex reflection group $W\left[\right.$ as to the symmetric group $\mathfrak{S}_{n}$.

Let $W$ be an irreducible complex reflection group of rank $n$ acting on its reflection representation $V \cong \mathbb{C}^{n}$. The action of $W$ on $V$ induces an action of $W$ on

- the dual space $V^{*}=\operatorname{Hom}_{\mathbb{C}}(V, \mathbb{C})$,

- the direct sum $V \oplus V^{*}$ of $V$ with its dual space, and finally

- the exterior algebra $\wedge\left(V \oplus V^{*}\right)$ over the $2 n$-dimensional vector space $V \oplus V^{*}$.

By placing $V$ in bidegree $(1,0)$ and $V^{*}$ in bidegree $(0,1)$, this last space $\wedge\left(V \oplus V^{*}\right)$ attains the structure of a doubly graded $W$-module.

If we let $\Theta_{n}=\left(\theta_{1}, \ldots, \theta_{n}\right)$ be a basis for $V$ and $\Xi_{n}=\left(\xi_{1}, \ldots, \xi_{n}\right)$ be a basis for $V^{*}$, we have a natural identification

$$
\wedge\left(V \oplus V^{*}\right)=\wedge\left\{\Theta_{n}, \Xi_{n}\right\}
$$

of $\wedge\left(V \oplus V^{*}\right)$ with the exterior algebra $\wedge\left\{\Theta_{n}, \Xi_{n}\right\}$ generated by the symbols $\theta_{i}$ and $\xi_{i}$ over $\mathbb{C}$. Following the terminology of physics, we refer to the $\theta_{i}$ and $\xi_{i}$ as fermionic variables. In physics, such variables are used to model fermions, with relations $\theta_{i}^{2}=\xi_{i}^{2}=0$ corresponding to the Pauli Exclusion Principle: no two fermions can occupy the same state at the same time. The model

Key words and phrases. coinvariant algebra, fermion, exterior algebra, Lefschetz element.

${ }^{1}$ And, in fact, to a wider class of groups $G$; see Remark 4.5 .

${ }^{2} \mathrm{~A}$ commuting variable $x_{i}$ is called bosonic; the power $x_{i}^{2}$ corresponds to two indistinguishable bosons in State $i$. 
$\wedge\left\{\Theta_{n}, \Xi_{n}\right\}$ for $\wedge\left(V \otimes V^{*}\right)$ will be helpful in our arguments. The following quotient ring is our object of study.

Definition 1.1. The fermionic diagonal coinvariant ring is the quotient

$$
D R_{W}:=\wedge\left(V \oplus V^{*}\right) /\left\langle\wedge\left(V \oplus V^{*}\right)_{+}^{W}\right\rangle
$$

of $\wedge\left(V \oplus V^{*}\right)$ by the (two-sided) ideal generated by the subspace $\wedge\left(V \oplus V^{*}\right)_{+}^{W} \subseteq \wedge\left(V \oplus V^{*}\right)$ of $W$-invariant elements with vanishing constant term.

The ideal $\left\langle\wedge\left(V \oplus V^{*}\right)_{+}^{W}\right\rangle$ is $W$-stable and bihomogeneous, so the quotient ring $D R_{W}$ has the structure of a bigraded $W$-module. We will see (Proposition 4.1) that this ideal is principal, generated by a 'Casimir element' $\delta_{W} \in V \oplus V^{*}$. Our results are as follows.

- We describe the bigraded $W$-isomorphism type of $D R_{W}$ in terms of the isomorphism types of the exterior powers $\wedge^{i} V$ and $\wedge^{j} V^{*}$ (Theorem 4.2).

- We show that $\operatorname{dim} D R_{W}=\left(\begin{array}{c}2 n+1 \\ n\end{array}\right)$ whenever $W$ has rank $n$ and relate the dimensions of its graded pieces to Catalan and Narayana numbers (Corollaries 4.3 and 4.4).

- We describe an explicit monomial basis of $D R_{W}$ using a variant of Motzkin paths (Theorem 5.2) and describe the bigraded Hilbert series of $D R_{W}$ in terms of the combinatorics of these paths (Corollary 5.4).

- When $W=\mathfrak{S}_{n}$, in Section [6 we give variants of the above results as they apply to the $n$-dimensional permutation representation of $\mathfrak{S}_{n}$ (as opposed to its $(n-1)$-dimensional reflection representation).

The key tool in our analysis is the realization (Theorem 3.2) of the Casimir generator $\delta_{W}$ of the ideal defining $D R_{W}$ as a kind of ' $W$-invariant Lefschetz element' in the ring $\wedge\left(V \oplus V^{*}\right)$. The ring $\wedge\left(V \oplus V^{*}\right)$, similar to the cohomology ring of a compact smooth complex manifold, satisfies 'bigraded' versions of Poincaré Duality and the Hard Lefschetz Theorem. This is somewhat unusual on two counts.

- Any homogeneous linear form in an exterior algebra squares to zero, and hence is not well-suited to be a (strong) Lefschetz element.

- Lefschetz elements arising in coinvariant theory are rarely $W$-invariant. For example, if $W$ is a Weyl group with associated complete flag manifold $G / B$ we may present the cohomology of $G / B$ as

$$
H^{\bullet}(G / B ; \mathbb{C})=\mathbb{C}[\mathfrak{h}] /\left\langle\mathbb{C}[\mathfrak{h}]_{+}^{W}\right\rangle
$$

where $\mathfrak{h}$ is the Cartan subalgebra of the Lie algebra $\mathfrak{g}$ of $G$. An element $\ell \in \mathbb{C}[\mathfrak{h}]_{1}=\mathfrak{h}^{*}$ is a Lefschetz element if and only if it is not fixed by any element of $W[13$. So the Lefschetz property is in some sense opposite to $W$-invariance in this case.

For examples of coinvariant-like quotients of superspace $\mathbb{C}[V] \otimes \wedge V^{*}$ satisfying other nontraditional bigraded versions of Poincaré Duality and (conjecturally) Hard Lefschetz, see [16.

The remainder of the paper is organized as follows. In Section 2 we give background material on complex reflection groups, Gröbner theory associated to exterior algebras, and the representation theory of $\mathfrak{S}_{n}$. In Section 3 we prove that $\wedge\left(V \oplus V^{*}\right)$ satisfies bigraded versions of the Hard Lefschetz Property and Poincaré Duality. This builds on work of Hara and Watanabe [11] showing that the incidence matrix between complementary ranks of the Boolean poset $B(n)$ is invertible. In Section 4 we apply these Lefschetz results to determine the bigraded $W$-structure of $D R_{W}$. In Section 5 we describe the standard monomial basis of $D R_{W}$ using lattice paths. In Section 6 we specialize to $W=\mathfrak{S}_{n}$ and translate our results to the setting of the permutation representation of $\mathfrak{S}_{n}$. We close in Section $\mathbf{7}$ with some open problems. 


\section{BACKGROUND}

2.1. Complex reflection groups. Let $V=\mathbb{C}^{n}$ be an $n$-dimensional complex vector space. An element $t \in \mathrm{GL}(V)=\mathrm{GL}_{n}(\mathbb{C})$ is a reflection if its fixed space $V^{t}:=\{v \in V: t(v)=v\}$ satisfies $\operatorname{dim} V^{t}=n-1$.

A finite subgroup $W \subseteq G L(V)$ is a complex reflection group if it is generated by reflections. The $W$-module $V$ is called the reflection representation of $W$. The $\operatorname{dimension} \operatorname{dim} V=n$ of $V$ is called the rank of $W$.

If $W_{1}$ and $W_{2}$ are reflection groups with reflection representations $V_{1}$ and $V_{2}$, the direct product $W_{1} \times W_{2}$ is naturally a reflection group with reflection representation $V_{1} \oplus V_{2}$. A reflection group $W$ acting on $V$ is irreducible if it is impossible to express $W$ as a direct product $W_{1} \times W_{2}$ of reflection groups acting on $V=V_{1} \oplus V_{2}$ unless $V_{1}=0$ or $V_{2}=0$.

2.2. Exterior Gröbner theory. Let $\Theta_{n}=\left(\theta_{1}, \ldots, \theta_{n}\right)$ be a list on $n$ anticommuting variables and let $\wedge\left\{\Theta_{n}\right\}$ be the exterior algebra generated by these variables over $\mathbb{C}$. For any subset $S=$ $\left\{i_{1}<i_{2}<\cdots<i_{k}\right\} \subseteq\{1,2, \ldots, n\}$, we let

$$
\theta_{S}:=\theta_{i_{1}} \theta_{i_{2}} \cdots \theta_{i_{k}}
$$

where the multiplication is in increasing order of subscripts. We refer to the $\theta_{S}$ as monomials; the set $\left\{\theta_{S}: S \subseteq\{1,2, \ldots, n\}\right\}$ is the monomial basis of $\wedge\left\{\Theta_{n}\right\}$. Given two monomials $\theta_{S}$ and $\theta_{T}$, we write $\theta_{S} \mid \theta_{T}$ to mean $S \subseteq T$.

A total order $<$ on the set $\left\{\theta_{S}: S \subseteq\{1,2, \ldots, n\}\right\}$ is a term order if

- we have $1=\theta_{\varnothing} \leq \theta_{S}$ for all $S$ and

- for all subsets $S, T, U$ with $U \cap S=U \cap T=\varnothing, \theta_{S}<\theta_{T}$ implies $\theta_{S \cup U}<\theta_{T \cup U}$.

Given a term order $<$, for any nonzero element $f \in \wedge\left\{\Theta_{n}\right\}$, let $\operatorname{LM}(f)$ be the largest monomial $\theta_{S}$ under the total order $<$ such that $\theta_{S}$ appears with nonzero coefficient in $f$. If $I \subseteq \wedge\left\{\Theta_{n}\right\}$ is a two-sided ideal, let

$$
\operatorname{LM}(I):=\{\operatorname{LM}(f): f \in I-\{0\}\}
$$

stand for the set of leading monomials of nonzero elements in $I$. The collection of standard monomials (or normal forms) for $I$ is

$$
N(I):=\left\{\text { monomials } \theta_{S}: S \subseteq\{1,2, \ldots, n\} \text { and } \theta_{S} \notin \operatorname{LM}(I)\right\} .
$$

The set $N(I)$ of monomials descends to a $\mathbb{C}$-basis of the quotient $\wedge\left\{\Theta_{n}\right\} / I$; this is the standard monomial basis with respect to $<$ (see for example [4]).

2.3. Representation Theory. If $V=\bigoplus_{i, j \geq 0} V_{i, j}$ is a bigraded vector space with each piece $V_{i, j}$ finite-dimensional, the bigraded Hilbert series is $\operatorname{Hilb}(V ; q, t):=\sum_{i, j \geq 0} \operatorname{dim} V_{i, j} \cdot q^{i} t^{j}$. This is a formal power series in $q$ and $t$.

The irreducible representations of the symmetric group $\mathfrak{S}_{n}$ are in one-to-one correspondence with partitions $\lambda \vdash n$. Given $\lambda \vdash n$, let $S^{\lambda}$ be the corresponding $\mathfrak{S}_{n}$-irreducible. For example, the trivial representation is $S^{(n)}$ and the sign representation is $S^{\left(1^{n}\right)}$.

Let $\Lambda$ denote the ring of symmetric functions and let $\left\{s_{\lambda}: \lambda\right.$ a partition $\}$ denote its Schur basis. The Hall inner product on $\Lambda$ declares the Schur basis to be orthonormal:

$$
\left\langle s_{\lambda}, s_{\mu}\right\rangle=\delta_{\lambda, \mu}
$$

for any partitions $\lambda$ and $\mu$.

Any finite-dimensional $\mathfrak{S}_{n}$-module $U$ may be expressed uniquely as a direct $\operatorname{sum} U \cong \bigoplus_{\lambda \vdash n} c_{\lambda} S^{\lambda}$ for some multiplicities $c_{\lambda}$. The Frobenius image of $U$ is the symmetric function

$$
\operatorname{Frob}(U):=\sum_{\lambda \vdash n} c_{\lambda} s_{\lambda},
$$


where $s_{\lambda}$ is the Schur function. The Kronecker product of two Schur functions $s_{\lambda}$ and $s_{\mu}$ for $\lambda, \mu \vdash n$ is defined by

$$
s_{\lambda} * s_{\mu}=\operatorname{Frob}\left(S^{\lambda} \otimes S^{\mu}\right)
$$

where $\mathfrak{S}_{n}$ acts diagonally on $S^{\lambda} \otimes S^{\mu}$.

If $V=\bigoplus_{i, j \geq 0} V_{i, j}$ is a bigraded $\mathfrak{S}_{n}$-module with each piece $V_{i, j}$ finite-dimensional, the bigraded Frobenius image is

$$
\operatorname{grFrob}(V ; q, t)=\sum_{i, j \geq 0} \operatorname{Frob}\left(V_{i, j}\right) \cdot q^{i} t^{j}
$$

This is a formal power series in $q$ and $t$ with coefficients in the ring of symmetric functions.

\section{LEFSCHETZ THEORY FOR EXTERIOR ALGEBRAS}

Let $A=\bigoplus_{i=0}^{n} A_{i}$ be a commutative graded $\mathbb{C}$-algebra. The algebra $A$ satisfies Poincaré Duality (PD) if $A_{n} \cong \mathbb{C}$ and if the multiplication map $A_{i} \otimes A_{n-i} \rightarrow A_{n} \cong \mathbb{C}$ is a perfect pairing for $0 \leq i \leq n$. In particular, this implies that the Hilbert series of $A$ is palindromic, i.e. $\operatorname{dim} A_{i}=\operatorname{dim} A_{n-i}$.

If $A=\bigoplus_{i=0}^{n} A_{i}$ satisifes $\mathrm{PD}$, an element $\ell \in A_{1}$ is called a (strong) Lefschetz element if for every $0 \leq i \leq n / 2$, the linear map

$$
\ell^{n-2 i} \cdot(-): A_{i} \rightarrow A_{n-i}
$$

given by multiplication by $\ell^{n-2 i}$ is bijective. If $A$ has a Lefschetz element, it is said to satisfy the Hard Lefschetz Property (HLP).

Algebras $A$ which satisfy PD and the HLP arise naturally in geometry as the cohomology rings (with adjusted grading) of smooth complex projective varieties. The HLP of the cohomology ring of the $n$-fold product $\mathbb{P}^{1} \times \cdots \times \mathbb{P}^{1}$ of 1 -dimensional complex projective space with itself was studied combinatorially by Hara and Watanabe [11]; we state their results below.

Recall that the Boolean poset $B(n)$ is the partial order on all subsets $S \subseteq\{1, \ldots, n\}$ given by $S \leq T$ if and only if $S \subseteq T$. The poset $B(n)$ is graded, with the $i^{t h}$ rank given by the family $B(n)_{i}$ of $i$-element subsets of $\{1, \ldots, n\}$. Hara and Watanabe proved that the incidence matrix between complementary ranks of $B(n)$ is invertible.

Theorem 3.1. (Hara-Watanabe [11]) Given $r \leq s \leq n$, define a $\left(\begin{array}{l}n \\ s\end{array}\right) \times\left(\begin{array}{l}n \\ r\end{array}\right)$ matrix $M_{n}(r, s)$ with rows indexed by $B(n)_{s}$ and columns indexed by $B(n)_{r}$ with entires

$$
M_{n}(r, s)_{T, S}= \begin{cases}1 & \text { if } S \subseteq T \\ 0 & \text { otherwise } .\end{cases}
$$

For any $0 \leq i \leq n$, the square matrix $M_{n}(i, n-i)$ is invertible.

For example, if $n=4$ and $i=1$, Theorem 3.1 asserts that the 0,1 -matrix $M_{4}(1,3)$ given by

$\{1\}$
$\{1,2,3\}$
$\{1,2,4\}$
$\{1,3,4\}$
$\{2,3,4\}$$\left(\begin{array}{cccc}1 & 1 & 13\} & \{4\} \\ 1 & 1 & 0 & 1 \\ 1 & 0 & 1 & 1 \\ 0 & 1 & 1 & 1\end{array}\right)$

is invertible.

The cohomology ring of the $n$-fold product $\mathbb{P}^{1} \times \cdots \times \mathbb{P}^{1}$ may be presented as

$$
H^{\bullet}\left(\mathbb{P}^{1} \times \cdots \times \mathbb{P}^{1} ; \mathbb{C}\right)=\mathbb{C}\left[x_{1}, \ldots, x_{n}\right] /\left\langle x_{1}^{2}, \ldots, x_{n}^{2}\right\rangle
$$


where $x_{i}$ represents the Chern class $c_{1}\left(\mathcal{L}_{i}\right)$ and $\mathcal{L}_{i}$ is the dual of the tautological line bundle over the $i^{\text {th }}$ factor of $\mathbb{P}^{1} \times \cdots \times \mathbb{P}^{1}$. Hara and Watanabe used Theorem 3.1 to give a combinatorial proof of the fact that $x_{1}+\cdots+x_{n}$ is a Lefschetz element for the $\operatorname{ring} H^{\bullet}\left(\mathbb{P}^{1} \times \cdots \times \mathbb{P}^{1} ; \mathbb{C}\right)[11$.

We want to study PD and the HLP in the context of the exterior algebra $\wedge\left\{\Theta_{n}, \Xi_{n}\right\}$. This algebra satisfies a natural bigraded version of Poincaré Duality: the top bidegree $\wedge\left\{\Theta_{n}, \Xi_{n}\right\}_{n, n}$ is 1-dimensional and the multiplication map

$$
\wedge\left\{\Theta_{n}, \Xi_{n}\right\}_{i, j} \otimes \wedge\left\{\Theta_{n}, \Xi_{n}\right\}_{n-i, n-j} \rightarrow \wedge\left\{\Theta_{n}, \Xi_{n}\right\}_{n, n} \cong \mathbb{C}
$$

is a perfect pairing for any $0 \leq i, j \leq n$.

The notion of a Lefschetz element in $\wedge\left\{\Theta_{n}, \Xi_{n}\right\}$ is a bit more subtle because any linear form $\ell$ in the variables $\theta_{1}, \ldots, \theta_{n}, \xi_{1}, \ldots, \xi_{n}$ satisfies $\ell^{2}=0$. To get around this, we introduce the element

$$
\delta_{n}:=\theta_{1} \xi_{1}+\theta_{2} \xi_{2}+\cdots+\theta_{n} \xi_{n} \in \wedge\left\{\Theta_{n}, \Xi_{n}\right\}_{1,1}
$$

The following result states that $\delta_{n}$ is a bigraded version of a Lefschetz element for the ring $\wedge\left\{\Theta_{n}, \Xi_{n}\right\}$.

Theorem 3.2. Suppose $i+j \leq n$ and let $r=n-i-j$. The linear map

$$
\varphi: \wedge\left\{\Theta_{n}, \Xi_{n}\right\}_{i, j} \stackrel{\delta_{n}^{r} .}{\longrightarrow} \wedge\left\{\Theta_{n}, \Xi_{n}\right\}_{n-j, n-i}
$$

given by multiplication by $\delta_{n}^{r}$ is bijective.

Proof. The idea is to introduce strategically chosen bases of the domain and target of $\varphi$ and show that the matrix representing $\varphi$ with respect to these bases is invertible using Theorem 3.1 .

Given two subsets $A, B \subseteq\{1, \ldots, n\}$, write

$$
\begin{aligned}
& A-B=\left\{a_{1}<a_{2}<\cdots<a_{r}\right\} \\
& B-A=\left\{b_{1}<b_{2}<\cdots<b_{s}\right\} \\
& A \cap B=\left\{c_{1}<c_{2}<\cdots<c_{t}\right\}
\end{aligned}
$$

and set

$$
\mathbf{v}(A, B):=\xi_{c_{1}} \theta_{c_{1}} \xi_{c_{2}} \theta_{c_{2}} \cdots \xi_{c_{t}} \theta_{c_{t}} \cdot \theta_{a_{1}} \theta_{a_{2}} \cdots \theta_{a_{r}} \cdot \xi_{b_{1}} \xi_{b_{2}} \cdots \xi_{b_{s}} .
$$

The family $\{\mathbf{v}(A, B): A, B \subseteq\{1, \ldots, n\}\}$ is a basis of $\wedge\left\{\Theta_{n}, \Xi_{n}\right\}$. For any sets $A$ and $B$, a direct computation shows

$$
\delta_{n} \cdot \mathbf{v}(A, B)=\sum_{c \notin A \cup B} \mathbf{v}(A \cup c, B \cup c) .
$$

The somewhat unusual variable order in the product $\mathbf{v}(A, B)$ was chosen strategically so that Equation (3.8) does not contain any signs.

Now suppose $|A|=i$ and $|B|=j$ for $i+j \leq n$ and set $r=n-i-j$. Iterating Equation (3.8) yields

$$
\delta_{n}^{r} \cdot \mathbf{v}(A, B)=\sum_{\substack{|C|=n-j,|D|=n-i \\ A \subseteq C, B \subseteq D|=r\\| C \cap D|-| A \cap B \mid=r}} \mathbf{v}(C, D) .
$$

We need to show that the (square) matrix of dimensions $\left(\begin{array}{c}n \\ i\end{array}\right) \cdot\left(\begin{array}{c}n \\ j\end{array}\right) \times\left(\begin{array}{c}n \\ n-j\end{array}\right) \cdot\left(\begin{array}{c}n \\ n-i\end{array}\right)$ defined by the system (3.9) is invertible. For any $\mathbf{v}(C, D)$ appearing in on the right-hand side of (3.9) we have $A-B=C-D=: I$ and $B-A=D-C=: J$. The matrix representing $\varphi$ therefore breaks up as a direct sum of smaller matrices indexed by the two sets $I$ and $J$, so we only need to show that every $(I, J)$-submatrix is invertible. 
For fixed $I$ and $J$, the submatrix in the previous paragraph is determined by the system

$$
\delta_{n}^{r} \cdot \mathbf{v}(A, B)=\sum_{\substack{T \subseteq S \\|T|=r}} r ! \cdot \mathbf{v}(A \cup T, B \cup T)
$$

where $S:=\{1, \ldots, n\}-(A \cup B)$. The system (3.10) represents a linear map

$$
\begin{aligned}
\operatorname{span}\{\mathbf{v}(A, B):|A|= & i,|B|=j, A-B=I, B-A=J\} \rightarrow \\
& \operatorname{span}\{\mathbf{v}(C, D):|C|=n-j,|D|=n-i, C-D=I, D-C=J\}
\end{aligned}
$$

where all sets $A, B, C, D$ are subsets of $\{1, \ldots, n\}$. In particular, the system (3.10) represents a square matrix of size $\left(\begin{array}{c}n-|I|-|J| \\ k\end{array}\right)$ where $k:=i-|I|=j-|J|$ is the size of the intersection $|A \cap B|$ for any element $\mathbf{v}(A, B)$ appearing in the LHS. If we let $S^{\prime}:=\{1, \ldots, n\}-(I \cup J)$, The invertibility of the system (3.10) is equivalent to the invertibility of the system

$$
\delta_{n}^{r} \cdot \mathbf{v}(R, R)=\sum_{\substack{T \subseteq S^{\prime} \\|T|=r}} r ! \cdot \mathbf{v}(R \cup T, R \cup T)
$$

representing a linear map

$$
\operatorname{span}\left\{\mathbf{v}(R, R): R \subseteq S^{\prime} \text { and }|R|=k\right\} \rightarrow \operatorname{span}\left\{\mathbf{v}\left(R^{\prime}, R^{\prime}\right): R^{\prime} \subseteq S^{\prime} \text { and }\left|R^{\prime}\right|=k+r\right\} .
$$

Observe that

$$
k+(k+r)=(i-|I|)+(j-|J|)+n-i-j=n-|I|-|J|=\left|S^{\prime}\right|
$$

so that the system (3.12) is invertible by Theorem 3.1 .

\section{Casimir Elements and Fermionic diagonal Coinvariants}

Let $W$ be an irreducible reflection group with reflection representation $V=\mathbb{C}^{n}$. Let $\theta_{1}, \ldots, \theta_{n}$ be a basis of $V$. Given the choice of $\theta_{1}, \ldots, \theta_{n}$, we let $\xi_{1}, \ldots, \xi_{n}$ be the dual basis of $V^{*}$ characterized by

$$
\xi_{i}\left(\theta_{j}\right)=\delta_{i, j}
$$

We rename the element $\delta_{n}=\theta_{1} \xi_{1}+\cdots+\theta_{n} \xi_{n}$ of $\wedge\left(V \oplus V^{*}\right)$ studied in the previous section as $\delta_{W}$ :

$$
\delta_{W}:=\delta_{n}=\theta_{1} \xi_{1}+\cdots+\theta_{n} \xi_{n} \in V \otimes V^{*} \subseteq \wedge\left(V \oplus V^{*}\right) .
$$

We refer to $\delta_{W}$ as the Casimir element of $W$.

The full general linear group $\mathrm{GL}(V)$ acts on $\wedge\left(V \oplus V^{*}\right)$ and it is not difficult to check using elementary matrices and the dual basis property that $\delta_{W}$ is invariant under this action. Equivalently, the Casimir element $\delta_{W}$ is independent of the choice of basis $\theta_{1}, \ldots, \theta_{n}$. In particular, the element $\delta_{W}$ lies in the $W$-invariant subring $\wedge\left(V \oplus V^{*}\right)^{W}$ of $\wedge\left(V \oplus V^{*}\right)$. In fact,

Proposition 4.1. The Casimir element $\delta_{W}$ generates the $W$-invariant subring $\wedge\left(V \oplus V^{*}\right)^{W}$ of $\wedge\left(V \oplus V^{*}\right)$.

Proof. Let $G$ be a finite group and let $U, U^{\prime}$ be irreducible complex representations of $G$. The tensor product $U \otimes U^{\prime}$ is a $G$-module by the rule $g .\left(u \otimes u^{\prime}\right):=(g . u) \otimes\left(g . u^{\prime}\right)$ for $g \in G, u \in U$, and $u^{\prime} \in U^{\prime}$. (This is the Kronecker product of the modules $U$ and $U^{\prime}$.) Character orthogonality implies multiplicity of the trivial $G$-module in $U \otimes U^{\prime}=\operatorname{dim}\left(U \otimes U^{\prime}\right)^{G}$

$$
= \begin{cases}1 & U^{\prime} \cong U^{*} \\ 0 & \text { otherwise. }\end{cases}
$$

Since $W$ is an irreducible complex reflection group, a result of Steinberg (see [12, Thm. A, $\S 24-3$, p. 250]) implies that the exterior powers $\wedge^{0} V, \wedge{ }^{1} V, \ldots, \wedge^{n} V$ are pairwise nonisomorphic 
irreducible representations of $W$. The same is true of their duals $\wedge^{0} V^{*}, \wedge^{1} V^{*}, \ldots, \wedge^{n} V^{*}$. Since the $(i, j)$-bidegree of $\wedge\left(V \oplus V^{*}\right)$ is given by $\wedge\left(V \oplus V^{*}\right)_{i, j}=\wedge^{i} V \otimes \wedge^{j} V^{*}$, the argument of the last paragraph gives

$$
\operatorname{dim} \wedge\left(V \oplus V^{*}\right)_{i, j}^{W}= \begin{cases}1 & i=j \\ 0 & i \neq j\end{cases}
$$

for any $0 \leq i, j \leq n$. On the other hand, we have $\delta_{W} \in \wedge\left(V \oplus V^{*}\right)_{1,1}^{W}$ and a quick computation shows that the powers $\delta_{W}^{0}, \delta_{W}^{1}, \ldots, \delta_{W}^{n}$ are nonzero. The proposition follows.

We are ready to describe the bigraded $W$-module structure of $D R_{W}$. We state our answer in terms of the Grothendieck ring of $W$. Recall that this is the $\mathbb{Z}$-algebra generated by the symbols $[U]$ where $U$ is a finite-dimensional $W$-module and subject to a relation $[U]=\left[U^{\prime}\right]+\left[U^{\prime \prime}\right]$ for any short exact sequence

$$
0 \rightarrow U^{\prime} \rightarrow U \rightarrow U^{\prime \prime} \rightarrow 0
$$

In particular, if $U \cong U^{\prime}$ we have $[U]=\left[U^{\prime}\right]$. Multiplication in the Grothendieck ring corresponds to Kronecker product, i.e. $[U] \cdot\left[U^{\prime}\right]:=\left[U \otimes U^{\prime}\right]$.

Theorem 4.2. Let $W$ be an irreducible complex reflection group acting on its reflection representation $V=\mathbb{C}^{n}$ and let $0 \leq i, j \leq n$. If $i+j>n$ we have $\left(D R_{W}\right)_{i, j}=0$. If $i+j \leq n$, inside the Grothendieck group of $W$ we have

$$
\left[\left(D R_{W}\right)_{i, j}\right]=\left[\wedge^{i} V\right] \cdot\left[\wedge^{j} V^{*}\right]-\left[\wedge^{i-1} V\right] \cdot\left[\wedge^{j-1} V^{*}\right]
$$

where we interpret $\wedge^{-1} V=\wedge^{-1} V^{*}=0$.

Proof. Thanks to Proposition 4.1 we can model $D R_{W}$ as

$$
D R_{W}=\wedge\left(V \oplus V^{*}\right) /\left\langle\delta_{W}\right\rangle=\wedge\left\{\Theta_{n}, \Xi_{n}\right\} /\left\langle\delta_{n}\right\rangle .
$$

If $i=0$ or $j=0$, the claim follows since $\delta_{W}$ lies in bidegree $(1,1)$, so assume $i, j>0$.

If $i+j \leq n$, let $r=n-i-j+1$. Theorem 3.2 implies that the multiplication map

$$
\delta_{W}^{r} \cdot(-): \wedge\left(V \oplus V^{*}\right)_{i-1, j-1} \rightarrow \wedge\left(V \oplus V^{*}\right)_{n-j+1, n-i+1}
$$

is a linear isomorphism. Whenever a composition $f \circ g$ of maps is a bijection, the map $g$ is injective and the map $f$ is surjective. Therefore, the map

$$
\delta_{W} \cdot(-): \wedge\left(V \oplus V^{*}\right)_{i-1, j-1} \rightarrow \wedge\left(V \oplus V^{*}\right)_{i, j}
$$

is an injection which we know to be $W$-equivariant. The claimed decomposition of $\left[\left(D R_{W}\right)_{i, j}\right]$ follows from (4.8).

Now suppose $i+j>n$. By analogous reasoning

$$
\delta_{W} \cdot(-): \wedge\left(V \oplus V^{*}\right)_{i-1, j-1} \rightarrow \wedge\left(V \oplus V^{*}\right)_{i, j}
$$

is a $W$-equivariant surjection so that $\left(D R_{W}\right)_{i, j}=0$.

Corollary 4.3. If $W$ has rank $n$, the vector space dimension of $D R_{W}$ is $\operatorname{dim} D R_{W}=\left(\begin{array}{c}2 n+1 \\ n\end{array}\right)$. 
Proof. Since $\operatorname{dim} \wedge{ }^{k} V=\operatorname{dim} \wedge{ }^{k} V^{*}=\left(\begin{array}{l}n \\ k\end{array}\right)$ where $V$ is the reflection representation of $W$, Theorem4.2 yields

$$
\begin{aligned}
\operatorname{dim} D R_{W} & =\sum_{k=0}^{n}\left(\begin{array}{l}
n \\
k
\end{array}\right)\left(\begin{array}{c}
n \\
n-k
\end{array}\right)+\sum_{j=1}^{n}\left(\begin{array}{c}
n \\
j-1
\end{array}\right)\left(\begin{array}{c}
n \\
n-j
\end{array}\right) \\
& =\left(\begin{array}{c}
2 n \\
n
\end{array}\right)+\left(\begin{array}{c}
2 n \\
n-1
\end{array}\right) \\
& =\left(\begin{array}{c}
2 n+1 \\
n
\end{array}\right)
\end{aligned}
$$

by the Pascal recursion.

Recall that the Catalan and Narayana numbers are given by

$$
\operatorname{Cat}(n):=\frac{1}{n+1}\left(\begin{array}{c}
2 n \\
n
\end{array}\right) \text { and } \operatorname{Nar}(n, k):=\frac{1}{n}\left(\begin{array}{l}
n \\
k
\end{array}\right)\left(\begin{array}{c}
n \\
k-1
\end{array}\right) .
$$

We have $\sum_{k=1}^{n} \operatorname{Nar}(n, k)=\operatorname{Cat}(n)$. These numbers have many combinatorial interpretations; for example, $\operatorname{Cat}(n)$ counts the number of Dyck paths of size $n$ and $\operatorname{Nar}(n, k)$ counts the number of such paths with $k-1$ peaks. The Catalan and Narayana numbers show up as the dimensions of the 'boundary' pieces of $D R_{W}$.

Corollary 4.4. If $W$ has rank $n$, we have

$$
\operatorname{dim}\left(D R_{W}\right)_{k, n-k}=\operatorname{Nar}(n+1, k+1)
$$

for $0 \leq k \leq n$ so that

$$
\sum_{k=0}^{n} \operatorname{dim}\left(D R_{W}\right)_{k, n-k}=\operatorname{Cat}(n+1) .
$$

Proof. Thanks to Theorem 4.2 one need only verify the identity

$$
\operatorname{Nar}(n+1, k+1)=\left(\begin{array}{l}
n \\
k
\end{array}\right)\left(\begin{array}{c}
n \\
n-k
\end{array}\right)-\left(\begin{array}{c}
n \\
k-1
\end{array}\right)\left(\begin{array}{c}
n \\
n-k-1
\end{array}\right),
$$

which is straightforward.

For any reflection group $W$, there are Catalan and Narayana numbers attached to $W$ (see for example [2]); the numbers appearing in Corollary 4.4 are their type A instances, and depend only on the rank of $W$.

Remark 4.5. The results in this section and the next apply equally well to any finite group $G$ and any $n$-dimensional $G$-module $V$ for which the exterior powers $\wedge \wedge^{0} V, \wedge{ }^{1} V, \ldots, \wedge \wedge^{n} V$ are pairwise nonisomorphic irreducibles. The proofs go through mutatis mutandis.

\section{MotZKin PATHS AND STANDARD BASES}

In this section we describe the standard monomial basis of $D R_{W}$ (with respect to a term order $\prec$ which we will define) in terms of a certain family of lattice paths. A Motzkin path is a lattice path in $\mathbb{Z}^{2}$ consisting of up-steps $(1,1)$, down-steps $(1,-1)$, and horizontal steps $(1,0)$ which starts at the origin, ends on the $x$-axis, and never sinks below the $x$-axis. We consider a variant of Motzkin paths which have decorated horizontal steps and need not end on the $x$-axis.

Let $\Pi(n)$ be the family of $n$-step lattice paths $\sigma=\left(s_{1}, \ldots, s_{n}\right)$ in $\mathbb{Z}^{2}$ which start at the origin and consist of up-steps $(1,1)$, down-steps $(1,-1)$, and horizontal steps $(1,0)$ in which each horizontal step is decorated with a $\theta$ or a $\xi$. We let $\Pi(n)_{\geq 0} \subseteq \Pi(n)$ be the family of paths which never sink below the $x$-axis. Two paths in $\Pi(9)$ are shown in Figure 1; the top path lies in $\Pi(9)_{\geq 0}$ but the bottom path does not. 


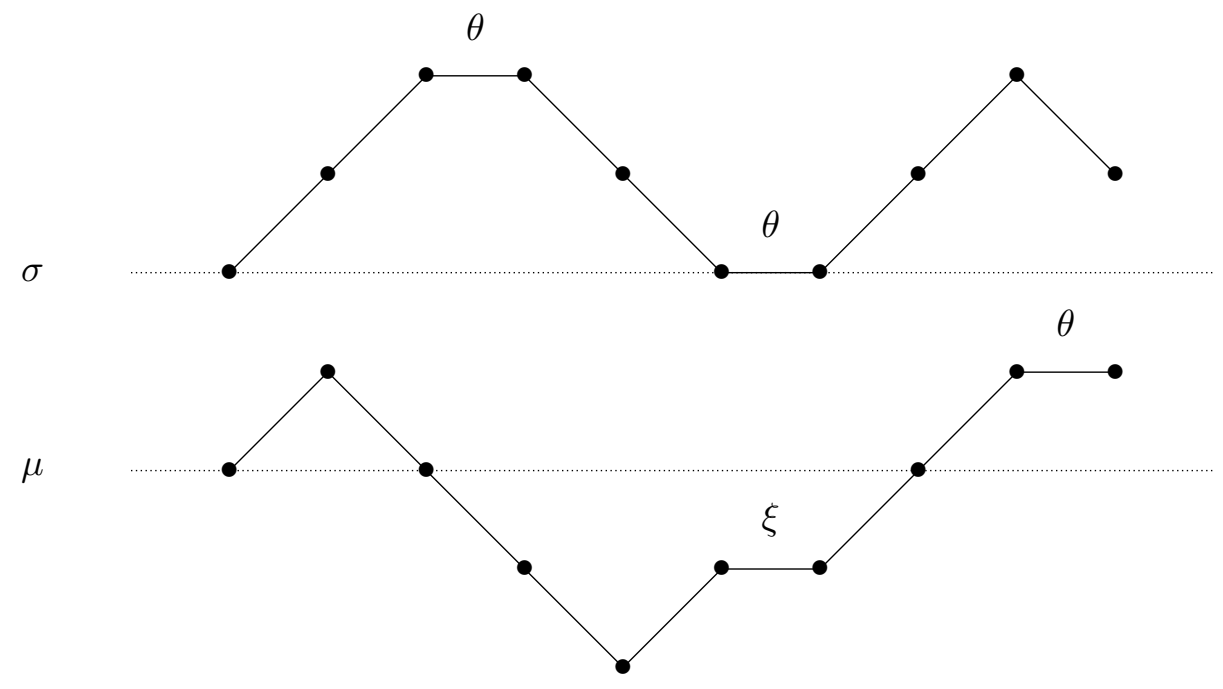

Figure 1. Two paths in $\Pi(9)$.

The depth $d(\sigma)$ of a path $\sigma \in \Pi(n)$ is the minimum $y$-value attained by $\sigma$. If $\sigma$ and $\mu$ are as in Figure 1 then $d(\sigma)=0$ and $d(\mu)=-2$. We have

$$
\Pi(n)_{\geq 0}=\{\sigma \in \Pi(n): d(\sigma)=0\}
$$

and $d(\sigma)<0$ for any $\sigma \in \Pi(n)-\Pi(n)_{\geq 0}$.

Let $\sigma=\left(s_{1}, \ldots, s_{n}\right) \in \Pi(n)$. The weight of the $i^{\text {th }}$ step $s_{i}$ of $\sigma$ is

$$
\operatorname{wt}(\sigma):= \begin{cases}1 & \text { if } s_{i}=(1,1) \text { is an up-step } \\ \theta_{i} & \text { if } s_{i}=(1,0) \text { is decorated with } \theta \\ \xi_{i} & \text { if } s_{i}=(1,0) \text { is decorated with } \xi \\ \theta_{i} \xi_{i} & \text { if } s_{i}=(1,-1) \text { is a down-step }\end{cases}
$$

and the weight of $\sigma$ is the product

$$
\operatorname{wt}(\sigma):=\operatorname{wt}\left(s_{1}\right) \cdots \operatorname{wt}\left(s_{n}\right)
$$

of the steps of $\sigma$ in the order in which they appear. For the paths $\sigma$ and $\mu$ in Figure 1 we have

$$
\operatorname{wt}(\sigma)=\theta_{3} \cdot \theta_{4} \xi_{4} \cdot \theta_{5} \xi_{5} \cdot \theta_{6} \cdot \theta_{9} \xi_{9} \quad \text { and } \quad \operatorname{wt}(\mu)=\theta_{2} \xi_{2} \cdot \theta_{3} \xi_{3} \cdot \theta_{4} \xi_{4} \cdot \xi_{6} \cdot \theta_{9} .
$$

A moment's thought shows that $\sigma \mapsto \operatorname{wt}(\sigma)$ gives a bijection from $\Pi(n)$ to the set of monomials in $\wedge\left\{\Theta_{n}, \Xi_{n}\right\}$, where monomials with differing signs are considered equivalent. We will identify paths $\sigma$ with their monomials wt $(\sigma)$.

The (total) degree of a path $\sigma$ is

$$
\operatorname{deg}(\sigma):=n-(\text { the terminal } y \text {-coordinate of } \sigma) .
$$

This is simply the total number of exterior generators $\theta_{i}$ and $\xi_{i}$ appearing in the monomial $\sigma$. We define the $\theta$-degree $\operatorname{deg}_{\theta}(\sigma)$ and $\xi$-degree $\operatorname{deg}_{\xi}(\sigma)$ analogously. Combinatorially,

$$
\operatorname{deg}_{\theta}(\sigma)=(\text { number of down-steps })+(\text { number of } \theta \text {-horizontal steps })
$$

and

$$
\left.\operatorname{deg}_{\xi}(\sigma)=\text { (number of down-steps }\right)+(\text { number of } \xi \text {-horizontal steps }) \text {. }
$$


If $\sigma$ and $\mu$ are as in Figure 1 then

$$
\left\{\begin{array} { l } 
{ \operatorname { d e g } ( \sigma ) = 8 } \\
{ \operatorname { d e g } _ { \theta } ( \sigma ) = 5 } \\
{ \operatorname { d e g } _ { \xi } ( \sigma ) = 3 }
\end{array} \quad \text { and } \quad \left\{\begin{array}{l}
\operatorname{deg}(\mu)=8 \\
\operatorname{deg}_{\theta}(\mu)=4 \\
\operatorname{deg}_{\xi}(\mu)=4
\end{array}\right.\right.
$$

We introduce the total order $\prec$ on paths $\sigma \in \Pi(n)$, or on monomials in $\wedge\left\{\Theta_{n}, \Xi_{n}\right\}$ given by

$$
\sigma \prec \sigma^{\prime} \Leftrightarrow \begin{cases}\operatorname{deg}(\sigma)<\operatorname{deg}\left(\sigma^{\prime}\right) & \text { or } \\ \operatorname{deg}(\sigma)=\operatorname{deg}\left(\sigma^{\prime}\right) \text { and } d(\sigma)>d\left(\sigma^{\prime}\right) & \text { or } \\ \operatorname{deg}(\sigma)=\operatorname{deg}\left(\sigma^{\prime}\right) \text { and } d(\sigma)=d\left(\sigma^{\prime}\right) \text { and } \sigma<_{\operatorname{lex}} \sigma^{\prime} & \end{cases}
$$

where in the last branch $<_{\text {lex }}$ means the lexicographical order on the paths $\sigma=\left(s_{1}, \ldots, s_{n}\right)$ and $\sigma^{\prime}=\left(s_{1}^{\prime}, \ldots, s_{n}^{\prime}\right)$ induced by declaring the step order

$$
(1,1)<(1,0) \text { with } \theta \text {-decoration }<(1,0) \text { with } \xi \text {-decoration }<(1,-1) .
$$

The collection of paths/monomials with a given bidegree $(i, j)$ form a subinterval of $\prec$ for all $0 \leq i, j \leq n$. In our running example of Figure 1, we have $\operatorname{deg}(\sigma)=\operatorname{deg}(\mu)$ but $d(\sigma)>d(\mu)$ so that $\sigma \prec \mu$.

Lemma 5.1. The total order $\prec i s$ a term order for $\wedge\left\{\Theta_{n}, \Xi_{n}\right\}$.

Proof. The first branch of the definition of $\prec$ guarantees that the monomial 1 with path consisting of a sequence of $n$ up-steps is the minimum monomial under $\prec$. Checking that $\prec$ respects multiplication amounts to the observation that total degree, depth, and lexicographical order are all respected by multiplication.

It turns out that the set $\left\{\operatorname{wt}(\sigma): \sigma \in \Pi(n)_{\geq 0}\right\}$ descends to a $\mathbb{C}$-basis of $D R_{W}$. In fact, we prove something stronger.

Theorem 5.2. The set $\left\{\operatorname{wt}(\sigma): \sigma \in \Pi(n)_{\geq 0}\right\}$ is the standard monomial basis of $D R_{W}$ with respect to $\prec$.

Proof. Let $I_{n}=\left\langle\delta_{n}\right\rangle \subseteq \wedge\left\{\Theta_{n}, \Xi_{n}\right\}$ be the defining ideal of $D R_{W}$ (here we apply Proposition 4.11). Identifying paths with monomials, we want to show $N\left(I_{n}\right)=\Pi(n)_{\geq 0}$ with respect to $\prec$. We proceed by induction on $n$, with the base case $n=1$ being immediate.

Suppose $n>1$ and $\sigma=\left(s_{1}, \ldots, s_{n}\right) \in \Pi(n)-\Pi(n)_{\geq 0}$. In particular, we have $d(\sigma)<0$. The following lemma will show inductively that $\sigma \notin N\left(I_{n}\right)$.

Lemma 5.3. The monomial $\sigma$ lies in $\operatorname{LM}\left(I_{n}\right)$ or else $\sigma=0$ in the quotient $D R_{W}$.

Proof. (of Lemma 5.3) Let $\sigma_{0} \in \wedge\left\{\Theta_{n-1}, \Xi_{n-1}\right\}$ be the monomial $\sigma$ with its last step $s_{n}$ removed. The proof breaks into cases depending on the step $s_{n}$.

Case 1: The last step $s_{n}$ is a horizontal step (of either decoration $\theta$ or $\xi$ ).

We assume the decoration of $s_{n}$ is $\theta$; the other case is similar. In this case, we have $\sigma_{0} \in$ $\Pi(n-1)-\Pi(n-1)_{\geq 0}$ and $\sigma=\sigma_{0} \theta_{n}$. We may inductively assume that $\sigma_{0} \in \operatorname{LM}\left(I_{n-1}\right)$ so that $\sigma_{0}=\operatorname{LM}\left(f \cdot \delta_{n-1}\right)$ for some polynomial $f \in \wedge\left\{\Theta_{n-1}, \Xi_{n-1}\right\}$. Since

$$
f \cdot \delta_{n} \cdot \theta_{n}=f \cdot \delta_{n-1} \theta_{n}+f \cdot \theta_{n} \xi_{n} \cdot \theta_{n}=f \cdot \delta_{n-1} \cdot \theta_{n},
$$

we conclude that $f \cdot \delta_{n-1} \cdot \theta_{n} \in I_{n}$. We have

$$
\operatorname{LM}\left(f \cdot \delta_{n-1} \cdot \theta_{n}\right)=\operatorname{LM}\left(f \cdot \delta_{n-1}\right) \cdot \theta_{n}=\sigma_{0} \cdot \theta_{n}=\sigma,
$$

completing the proof of Case 1.

Case 2: The last step $s_{n}$ is a down-step $(1,-1)$.

If $\sigma_{0} \in \Pi(n-1)-\Pi(n-1)_{\geq 0}$ sinks below the $x$-axis, the proof is similar to that of Case 1 . One right-multiplies $f \cdot \delta_{n}$ by $\theta_{n} \xi_{n}$ instead of $\theta_{n}$; we leave the details to the reader. 
In this case we could have $\sigma_{0} \in \Pi(n-1)_{\geq 0}$, but this would imply that $\sigma_{0}$ ends on the $x$-axis, so that $\operatorname{deg}(\sigma)=\operatorname{deg}\left(\sigma_{0}\right)+2=(n-1)+2=n+1$. Theorem 4.2 then forces $\sigma=0$ in the quotient $D R_{W}$, completing the proof of Case 2 .

Case 3: The last step $s_{n}$ is an up-step $(1,1)$.

This is the most involved case. We have $\sigma_{0}=\sigma$ and $\sigma_{0} \in \Pi(n-1)-\Pi(n-1)_{\geq 0}$. By induction, we may assume that there is $f \in \wedge\left\{\Theta_{n-1}, \Xi_{n-1}\right\}$ with $\sigma_{0}=\operatorname{LM}\left(f \cdot \delta_{n-1}\right)$. Now consider

$$
f \cdot \delta_{n}=f \cdot \delta_{n-1}+f \cdot \theta_{n} \xi_{n} \in I_{n} .
$$

By discarding redundant terms if necessary, we may assume that $f$ is bi-homogeneous. The monomial $\sigma=\sigma_{0}$ is the $\prec$-largest monomial appearing in $f \cdot \delta_{n-1}$. Since $\sigma$ does not involve $\theta_{n}$ or $\xi_{n}$, it does not appear in $f \cdot \theta_{n} \xi_{n}$. We will have $\sigma=\operatorname{LM}\left(f \cdot \delta_{n}\right)$ unless some monomial $\mu$ appearing in $f \cdot \theta_{n} \xi_{n}$ satisfies $\mu \succ \sigma$.

Let $\mu$ be the $\prec$-largest element of $f \cdot \theta_{n} \xi_{n}$ and assume $\sigma \prec \mu$. Let $\mu_{0} \in \Pi(n-1)$ be the path obtained from $\mu$ by removing its last step (which is necessarily a down-step since $\mu$ appears in $\left.f \cdot \theta_{n} \xi_{n}\right)$. Since $\sigma \prec \mu$, the bihomogeneity of $f$ forces $d(\mu) \leq d(\sigma)<0$.

Subcase 3.1: We have $\mu_{0} \in \Pi(n-1)_{\geq 0}$, or equivalently $d\left(\mu_{0}\right) \geq 0$.

Since $d(\mu)=d\left(\mu_{0}\right)+1<0$, this can only happen if $d\left(\mu_{0}\right)=0$ and $\mu_{0}$ ends at the lattice points $(n-1,0)$. This implies that $\operatorname{deg}(\mu)=\operatorname{deg}\left(\mu_{0}\right)+2=(n-1)+2=n+1$ and Theorem 4.2 forces $\mu \in I_{n}$. We may therefore discard the term involving $\mu$ from (5.13) and still have an element of $I_{n}$ involving $\sigma$.

Subcase 3.2: We have $\mu_{0} \in \Pi(n-1)-\Pi(n-1)_{\geq 0}$, or equivalently $d\left(\mu_{0}\right)<0$.

In this case, we induct on $n$ to obtain some polynomial $g \in \wedge\left\{\Theta_{n-1}, \Xi_{n-1}\right\}$ whose leading monomial is $\mu_{0}=\operatorname{LM}\left(g \cdot \delta_{n-1}\right)$. We calculate

$$
\operatorname{LM}\left(g \cdot \delta_{n} \cdot \theta_{n} \xi_{n}\right)=\operatorname{LM}\left(g \cdot \delta_{n-1} \cdot \theta_{n} \xi_{n}\right)=\operatorname{LM}\left(g \cdot \delta_{n-1}\right) \cdot \theta_{n} \xi_{n}=\mu_{0} \cdot \theta_{n} \xi_{n}=\mu
$$

where the second equality used the fact that $g \cdot \delta_{n-1}$ does not involve $\theta_{n}$ or $\xi_{n}$. Since $\sigma$ does not involve $\theta_{n}$ or $\xi_{n}$, it does not appear in $g \cdot \delta_{n} \cdot \theta_{n} \xi_{n}$. We may therefore replace (5.13) by

$$
f \cdot \delta_{n-1}+\left(f-g \cdot \delta_{n-1}\right) \cdot \theta_{n} \xi_{n} \in I_{n}
$$

to obtain another element of $I_{n}$ which involves $\sigma$ only in its first term, still satisfies $\sigma=\operatorname{LM}\left(f \cdot \delta_{n-1}\right)$, but now only involves monomials $\prec \mu$.

Iterating the arguments of Subcases 3.1 and 3.2, we see that $\sigma \in \operatorname{LM}\left(I_{n}\right)$, proving both Case 3 and the lemma.

We complete the proof of Theorem 5.2 using Lemma 5.3. Lemma 5.3 implies $N\left(I_{n}\right) \subseteq \Pi(n)_{\geq 0}$, and to force equality it suffices to verify

$$
\operatorname{dim} D R_{W}=\left|\Pi(n)_{\geq 0}\right| .
$$

In fact, we verify the equality of bigraded Hilbert series

$$
\operatorname{Hilb}\left(D R_{W} ; q, t\right)=\sum_{\sigma \in \Pi(n) \geq 0} q^{\operatorname{deg}_{\theta}(\sigma)} t^{\operatorname{deg}_{\xi}(\sigma)}=: P_{n}(q, t) .
$$

If we let $\Pi(n)_{=0} \subseteq \Pi(n)_{\geq 0}$ be the subset of paths that end on the $x$-axis and let

$$
P_{n}^{\prime}(q, t):=\sum_{\sigma \in \Pi(n)=0} q^{\operatorname{deg}_{\theta}(\sigma)} t^{\operatorname{deg}_{\xi}(\sigma)},
$$

considering the addition of one more step to a path yields

$$
P_{n+1}(q, t)=(1+q+t+q t) \cdot P_{n}(q, t)-(q t) \cdot P_{n}^{\prime}(q, t) .
$$


On the other hand (adopting the notation $D R_{W(n)}$ for $D R_{W}$ whenever $W$ has rank $n$ ) Theorem 4.2 yields

$$
\operatorname{dim}\left(D R_{W(n+1)}\right)_{i, j}= \begin{cases}\left(\begin{array}{c}
n+1 \\
i
\end{array}\right) \cdot\left(\begin{array}{c}
n+1 \\
j
\end{array}\right)-\left(\begin{array}{c}
n+1 \\
i-1
\end{array}\right) \cdot\left(\begin{array}{c}
n+1 \\
j-1
\end{array}\right) & \text { if } i, j>0 \text { and } i+j \leq n+1 \\
\left(\begin{array}{c}
n+1 \\
i
\end{array}\right) \cdot\left(\begin{array}{c}
n+1 \\
j
\end{array}\right) & \text { if } i=0 \text { or } j=0 \\
0 & \text { if } i+j>n+1\end{cases}
$$

It can be shown using the Pascal identity and Equation (5.20) that

$$
\operatorname{Hilb}\left(D R_{W(n+1)} ; q, t\right)=(1+q+t+q t) \cdot \operatorname{Hilb}\left(D R_{W(n)} ; q, t\right)-(q t) \cdot \sum_{i+j=n+1} \operatorname{dim}\left(D R_{W(n)}\right)_{i, j} \cdot q^{i} t^{j}
$$

which matches the combinatorial recursion in Equation (5.19).

In the course of proving Theorem 5.2, we derived the following combinatorial expression for the bigraded Hilbert series of $D R_{W}$.

Corollary 5.4. If $W$ has rank $n$, we have

$$
\operatorname{Hilb}\left(D R_{W} ; q, t\right)=\sum_{\sigma \in \Pi(n) \geq 0} q^{\operatorname{deg}_{\theta}(\sigma)} t^{\operatorname{deg}_{\xi}(\sigma)} .
$$

\section{The Permutation Representation of $\mathfrak{S}_{n}$}

In the coinvariant theory of the symmetric group $\mathfrak{S}_{n}$, it is more common to consider its $n$ dimensional permutation representation $U$ as opposed to its $(n-1)$-dimensional reflection representation $V$ In this section we describe how to translate our results into this setting.

The following decompositions of $U$ and $U^{*}$ into $\mathfrak{S}_{n}$-irreducibles are well-known:

$$
U=V \oplus U^{\mathfrak{S}_{n}} \quad \text { and } \quad U^{*}=V^{*} \oplus\left(U^{*}\right)^{\mathfrak{S}_{n}} .
$$

It follows that

$$
\begin{aligned}
\wedge\left(U \oplus U^{*}\right) & \cong \wedge\left[\left(V \oplus U^{\mathfrak{S}_{n}}\right) \oplus\left(V^{*} \oplus\left(U^{*}\right)^{\mathfrak{S}_{n}}\right)\right] \\
& \cong \wedge\left[\left(V \oplus V^{*}\right) \oplus\left(U^{\mathfrak{S}_{n}} \oplus\left(U^{*}\right)^{\mathfrak{S}_{n}}\right)\right] \\
& \cong\left[\wedge\left(V \oplus V^{*}\right)\right] \otimes\left[\wedge\left(U^{\mathfrak{S}_{n}} \oplus\left(U^{*}\right)^{\mathfrak{S}_{n}}\right)\right] .
\end{aligned}
$$

Modding out by ideals generated by $\mathfrak{S}_{n}$-invariants with vanishing constant term, we see that

$$
\wedge\left(U \otimes U^{*}\right) /\left\langle\wedge\left(U \otimes U^{*}\right)_{+}^{\mathfrak{S}_{n}}\right\rangle \cong \wedge\left(V \otimes V^{*}\right) /\left\langle\wedge\left(V \otimes V^{*}\right)_{+}^{\mathfrak{S}_{n}}\right\rangle
$$

Let $\mathfrak{S}_{n}$ act on $\wedge\left\{\Theta_{n}, \Xi_{n}\right\}$ diagonally, viz. $w \cdot \theta_{i}:=\theta_{w(i)}$ and $w \cdot \xi_{i}:=\xi_{w(i)}$. Expressing the left-hand side of (6.5) in terms of coordinates, we have the following translation of Theorem 4.2 , Corollary 4.3, and Corollary 4.3 .

Theorem 6.1. Let $D R_{n}$ be the bigraded $\mathfrak{S}_{n}$-module

$$
D R_{n}:=\wedge\left\{\Theta_{n}, \Xi_{n}\right\} /\left\langle\wedge\left\{\Theta_{n}, \Xi_{n}\right\}_{+}^{\mathfrak{S}_{n}}\right\rangle .
$$

We have $\left(D R_{n}\right)_{i, j}=0$ whenever $i+j \geq n$. If $i+j<n$, we have

$$
\operatorname{Frob}\left(D R_{n}\right)_{i, j}=s_{\left(n-i, 1^{i}\right)} * s_{\left(n-j, 1^{j}\right)}-s_{\left(n-i+1,1^{i-1}\right)} * s_{\left(n-j+1,1^{j-1}\right)}
$$

where $*$ denotes Kronecker product. Here we interpret $s_{(n+1,-1)}=0$. We have

$$
\operatorname{dim} D R_{n}=\left(\begin{array}{c}
2 n-1 \\
n
\end{array}\right)
$$

and, for $1 \leq k \leq n$, we have

$$
\operatorname{dim}\left(D R_{n}\right)_{k-1, n-k}=\operatorname{Nar}(n, k)
$$

so that $\sum_{k=1}^{n} \operatorname{dim}\left(D R_{n}\right)_{k-1, n-k}=\operatorname{Cat}(n)$. 
Equation (6.8) was conjectured by Mike Zabrocki [20, for the Open Problems in Algebraic Combinatorics 2020 Conference 3 . We also have a lattice path basis of the $\mathfrak{S}_{n}$-module $D R_{n}$ in Theorem 6.1. For a partition $\lambda \vdash n$, work of Rosas [17] implies that

$$
\left\langle\operatorname{grFrob}\left(D R_{n} ; q, t\right), s_{\lambda}\right\rangle=0
$$

unless the partition $\lambda=\left(\lambda_{1} \geq \lambda_{2} \geq \lambda_{3} \geq \cdots\right)$ satisfies $\lambda_{3} \leq 2$ (i.e. the Young diagram of $\lambda$ is a union of two possibly empty hooks). While these multiplicities can be less than aesthetic in general, they are nice when $\lambda$ is a hook. Recall that the $q, t$-analog of $n$ is given by

$$
[n]_{q, t}:=\frac{q^{n}-t^{n}}{q-t}=q^{n-1}+q^{n-2} t+\cdots+q t^{n-2}+t^{n-1} .
$$

Proposition 6.2. The graded multiplicities of the trivial and sign representations in $D R_{n}$ are given by

$$
\left\langle\operatorname{grFrob}\left(D R_{n} ; q, t\right), s_{(n)}\right\rangle=1 \quad \text { and } \quad\left\langle\operatorname{grFrob}\left(D R_{n} ; q, t\right), s_{\left(1^{n}\right)}\right\rangle=[n]_{q, t} .
$$

If $0<k<n-1$ we have

$$
\left\langle\operatorname{grFrob}\left(D R_{n} ; q, t\right), s_{\left(n-k, 1^{k}\right)}\right\rangle=[k+1]_{q, t}+(q t) \cdot[k]_{q, t} .
$$

Proof. The equation $\left\langle\operatorname{grFrob}\left(D R_{n} ; q, t\right), s_{(n)}\right\rangle=1$ is immediate since $D R_{n}$ is obtained from $\wedge\left\{\Theta_{n}, \Xi_{n}\right\}$ by modding out by $\mathfrak{S}_{n}$-invariants with vanishing constant term. The multiplicity of the signed representation follows from Theorem 6.1 and the fact that for any partitions $\lambda, \mu \vdash n$

$$
\text { multiplicity of } s_{\left(1^{n}\right)} \text { in } s_{\lambda} * s_{\mu}= \begin{cases}1 & \text { if } \mu=\lambda^{\prime} \\ 0 & \text { otherwise }\end{cases}
$$

where $\lambda^{\prime}$ is the conjugate (transpose) partition of $\lambda$.

We turn our attention to Equation (6.13). For any statement $P$, let $\chi(P)=1$ if $P$ is true and $\chi(P)=0$ if $P$ is false. Rosas proves [17, Proof of Thm. $13(4)$ ] that the multiplicity of the Schur function $s_{\left(n-c, 1^{c}\right)}$ in the Kronecker product $s_{\left(n-a, 1^{a}\right)} * s_{\left(n-b, 1^{b}\right)}$ is

$$
\left\langle s_{\left(n-a, 1^{a}\right)} * s_{\left(n-b, 1^{b}\right)}, s_{\left(n-c, 1^{c}\right)}\right\rangle=\chi(|b-a| \leq c) \times \chi(c \leq a+b \leq 2 n-c-2)
$$

whenever $0<a, b<n$ and $0<c<n-1$.

For any $0 \leq k \leq n-1$ and all $i+j<n$, we have

$$
\begin{aligned}
& \left\langle\operatorname{Frob}\left(D R_{n}\right)_{i, j}, s_{\left(n-k, 1^{k}\right)}\right\rangle= \\
& \quad\left\langle s_{\left(n-i, 1^{i}\right)} * s_{\left(n-j, 1^{j}\right)}, s_{\left(n-k, 1^{k}\right)}\right\rangle-\left\langle s_{\left(n-i+1,1^{i-1}\right)} * s_{\left(n-j+1,1^{j-1}\right)}, s_{\left(n-k, 1^{k}\right)}\right\rangle
\end{aligned}
$$

A somewhat tedious casework using Equation (6.15) yields

$$
\left\langle\operatorname{Frob}\left(D R_{n}\right)_{i, j}, s_{\left(n-k, 1^{k}\right)}\right\rangle= \begin{cases}1 & \text { if } i+j=k \\ 1 & \text { if } i+j=k+1 \text { and } i, j>0 \\ 0 & \text { otherwise }\end{cases}
$$

which is equivalent to Equation (6.13).

In order to state a $D R_{n}$-analog of Theorem 5.2, we need some notation. We define the primed weight $\mathrm{wt}^{\prime}(s)$ of a step $s$ to be

$$
\begin{cases}1 & \text { if } s=(1,1) \text { is an up-step } \\ \theta_{i} & \text { if } s=(1,0) \text { is decorated with } \theta \\ \xi_{i}^{\prime} & \text { if } s=(1,0) \text { is decorated with } \xi \\ \theta_{i} \xi_{i}^{\prime} & \text { if } s=(1,-1) \text { is a down-step }\end{cases}
$$

\footnotetext{
3 which was delayed due to COVID-19, but will hopefully take place soon
} 
where

$$
\xi_{i}^{\prime}:=\xi_{i}+\sum_{j=2}^{n} \xi_{j} .
$$

The primed weight $\mathrm{wt}^{\prime}(\sigma)$ of a path $\sigma \in \Pi(n)$ with steps $\sigma=\left(s_{1}, \ldots, s_{n}\right)$ is $\mathrm{wt}^{\prime}(\sigma):=\mathrm{wt}^{\prime}\left(s_{1}\right) \cdots \mathrm{wt}^{\prime}\left(s_{n}\right)$. Let $\Pi(n)_{>0} \subseteq \Pi(n)$ consist of those paths which only meet the $x$-axis at their starting point $(0,0)$ and stay strictly above the $x$-axis otherwise.

Theorem 6.3. The set $\left\{\operatorname{wt}^{\prime}(\sigma): \sigma \in \Pi(n)_{>0}\right\}$ descends to a basis of $D R_{n}$. Consequently, we have

$$
\operatorname{Hilb}\left(D R_{n} ; q, t\right)=\sum_{\sigma \in \Pi(n)>0} q^{\operatorname{deg}_{\theta}(\sigma)} t^{\operatorname{deg}_{\xi}(\sigma)} .
$$

Proof. Proposition 4.1 and the discussion prior to Theorem 6.1 imply that the invariant subalgebra $\wedge\left\{\Theta_{n}, \Xi_{n}\right\}^{\mathfrak{S}_{n}}$ is generated by the three elements

$$
\theta_{1}+\cdots+\theta_{n}, \xi_{1}+\cdots+\xi_{n}, \text { and } \theta_{1} \xi_{1}+\cdots+\theta_{n} \xi_{n}
$$

and consequently

$$
D R_{n}=\wedge\left\{\theta_{1}, \ldots, \theta_{n}, \xi_{1}, \ldots, \xi_{n}\right\} /\left\langle\theta_{1}+\cdots+\theta_{n}, \xi_{1}+\cdots+\xi_{n}, \theta_{1} \xi_{1}+\cdots+\theta_{n} \xi_{n}\right\rangle .
$$

We express $D R_{n}$ as a successive quotient

$$
\begin{aligned}
D R_{n} & =\wedge\left\{\theta_{1}, \ldots, \theta_{n}, \xi_{1}, \ldots, \xi_{n}\right\} /\left\langle\theta_{1}+\cdots+\theta_{n}, \xi_{1}+\cdots+\xi_{n}, \theta_{1} \xi_{1}+\cdots+\theta_{n} \xi_{n}\right\rangle . \\
& =\left(\wedge\left\{\theta_{1}, \ldots, \theta_{n}\right\} /\left\langle\sum_{i=1}^{n} \theta_{i}\right\rangle \otimes \wedge\left\{\xi_{1}, \ldots, \xi_{n}\right\} /\left\langle\sum_{i=1}^{n} \xi_{i}\right\rangle\right) /\left\langle\sum_{i=1}^{n} \theta_{i} \otimes \xi_{i}\right\rangle
\end{aligned}
$$

Then as graded vector spaces, we identify $\theta_{1}=-\theta_{2}-\cdots-\theta_{n}$ and $\xi_{1}=-\xi_{2}-\cdots-\xi_{n}$ to obtain

$$
\begin{aligned}
D R_{n} & \cong\left(\wedge\left\{\theta_{2}, \ldots, \theta_{n}\right\} \otimes \wedge\left\{\xi_{2}, \ldots, \xi_{n}\right\}\right) /\left\langle\left(-\theta_{2}-\cdots-\theta_{n}\right) \otimes\left(-\xi_{2}-\cdots-\xi_{n}\right)+\sum_{i=2}^{n} \theta_{i} \otimes \xi_{i}\right\rangle \\
& =\left(\wedge\left\{\theta_{2}, \ldots, \theta_{n}\right\} \otimes \wedge\left\{\xi_{2}, \ldots, \xi_{n}\right\}\right) /\left\langle\sum_{i=2}^{n} \theta_{i} \otimes\left(\xi_{i}+\sum_{j=2}^{n} \xi_{j}\right)\right\rangle
\end{aligned}
$$

The transition matrix from the set $\left\{\xi_{2}+\sum_{j=2}^{n} \xi_{j}, \ldots, \xi_{n}+\sum_{j=2}^{n} \xi_{j}\right\}=\left\{\xi_{2}^{\prime}, \ldots, \xi_{n}^{\prime}\right\}$ to the standard basis $\left\{\xi_{2}, \ldots, \xi_{n}\right\}$ of the degree 1 component of $\wedge\left\{\xi_{2}, \ldots, \xi_{n}\right\}$ is

$$
\left(\begin{array}{cccc}
2 & 1 & \cdots & 1 \\
1 & 2 & \cdots & 1 \\
\vdots & \vdots & \ddots & \vdots \\
1 & 1 & \cdots & 2
\end{array}\right)
$$

which is easily checked to be invertible. Therefore, the set $\left\{\xi_{2}^{\prime}, \ldots, \xi_{n}^{\prime}\right\}$ is also a basis of the degree 1 component of $\wedge\left\{\xi_{2}, \ldots, \xi_{n}\right\}$ and we may write

$$
D R_{n} \cong \wedge\left\{\theta_{2}, \ldots, \theta_{n}, \xi_{2}^{\prime}, \ldots, \xi_{n}^{\prime}\right\} /\left\langle\theta_{2} \xi_{2}^{\prime}+\cdots+\theta_{n} \xi_{n}^{\prime}\right\rangle .
$$

Theorem 5.2 applies to complete the proof. 


\section{Open Problems}

The key result underpinning our analysis of $D R_{W}$ and $D R_{n}$ was the Lefschetz Theorem 3.2. Our proof was combinatorial and ultimately relied on the Boolean poset $B(n)$. Given the importance of Lefschetz elements in geometry, it is natural to ask the following.

Question 7.1. Is there a geometric proof of Theorem 3.2]?

Modern variants of the HLP and PD were used to great effect in the work of Adiprasito, Huh, and Katz on the Chow rings of matroids [1]. Is there a deeper meaning to the HLP and PD as they apply to exterior algebras? Perhaps the realization of $\wedge\left\{\Theta_{n}, \Xi_{n}\right\}$ as the holomorphic tangent space to the origin in $\mathbb{C}^{n} \oplus \mathbb{C}^{n}$ would be relevant here.

It may also be interesting to consider combining two sets of commuting and anticommuting variables to get a ring

$$
\mathbb{C}\left[X_{n}, Y_{n}\right] \otimes \wedge\left\{\Theta_{n}, \Xi_{n}\right\}:=\mathbb{C}\left[x_{1}, \ldots, x_{n}, y_{1}, \ldots, y_{n}\right] \otimes \wedge\left\{\theta_{1}, \ldots, \theta_{n}, \xi_{1}, \ldots, \xi_{n}\right\}
$$

which may be identified with the algebra of polynomial-valued holomorphic differential forms on $\mathbb{C}^{n} \oplus \mathbb{C}^{n}$. This ring is quadruply graded, and the diagonal action of $\mathfrak{S}_{n}$ gives rise to a coinvariant space $\mathbb{C}\left[X_{n}, Y_{n}, \Theta_{n}, \Xi_{n}\right] /\left\langle\mathbb{C}\left[X_{n}, Y_{n}, \Theta_{n}, \Xi_{n}\right]_{+}^{\mathfrak{S}_{n}}\right\rangle$. Setting the $\xi$-variables to zero, Zabrocki [19] conjectured that the triply graded Frobenius image of this quotient is given by the Delta Conjecture of Haglund, Remmel, and Wilson [7. Furthermore, again when the $\xi$-variables are set to zero, Haglund and Sergel [9] have a conjectural monomial basis of this quotient which would extend a basis of the diagonal coinvariants due to Carlsson and Oblomkov [6].

Problem 7.2. Find a basis of the quotient $\mathbb{C}\left[X_{n}, Y_{n}, \Theta_{n}, \Xi_{n}\right] /\left\langle\mathbb{C}\left[X_{n}, Y_{n}, \Theta_{n}, \Xi_{n}\right]_{+}^{\mathfrak{S}_{n}}\right\rangle$ which generalizes the basis of $\mathbb{C}\left[X_{n}, Y_{n}\right] /\left\langle\mathbb{C}\left[X_{n}, Y_{n}\right]_{+}^{\mathfrak{S}_{n}}\right\rangle$ due to Carlsson-Oblomkov [6] and the conjectural basis of $\mathbb{C}\left[X_{n}, Y_{n}, \Theta_{n}\right] /\left\langle\mathbb{C}\left[X_{n}, Y_{n}, \Theta_{n}\right]_{+}^{\mathfrak{S}_{n}}\right\rangle$ due to Haglund-Sergel [9].

A solution to Problem 7.2 might be obtained by interpolating between the parking function 'schedules' present in [6, 9] and our Motzkin-like paths $\Pi(n)_{>0}$.

Let $X_{k \times n}=\left(x_{i, j}\right)_{1 \leq i \leq k, 1 \leq j \leq n}$ be a $k \times n$ matrix of commuting variables and let $\mathbb{C}\left[X_{k \times n}\right]$ be the polynomial ring in these variables. The ring $\mathbb{C}\left[X_{k \times n}\right]$ carries a $\mathfrak{S}_{n}$-module structure inherited from column permutation and the quotient $\mathbb{C}\left[X_{k \times n}\right] /\left\langle\mathbb{C}\left[X_{k \times n}\right]_{+}^{\mathfrak{S}_{n}}\right\rangle$ is a $\left(\mathbb{Z}_{>0}\right)^{k}$-graded $\mathfrak{S}_{n}$-module. When $k=2$, we recover the classical diagonal coinvariants. F. Bergeron has many fascinating conjectures about this object obtained by letting the parameter $k$ grow 3 .

We can carry out the contstruction of the previous paragraph with a matrix $\Theta_{k \times n}=\left(\theta_{i, j}\right)_{1 \leq i \leq k, 1 \leq j \leq n}$ of anticommuting variables. We still have an action of $\mathfrak{S}_{n}$ on columns and can still consider the quotient

$$
R(k \times n):=\wedge\left\{\Theta_{k \times n}\right\} /\left\langle\wedge\left\{\Theta_{k \times n}\right\}_{+}^{\mathfrak{S}_{n}}\right\rangle .
$$

In the case $k=2$ we recover $D R_{n}$. For stability results involving such quotients, and corresponding quotients using both commuting and anticommuting variables, see [15].

Question 7.3. Find the multigraded isomorphism type of $R(k \times n)$.

It is unclear how to use Lefschetz Theory to solve Question 7.3 for $k>2$. For any set $S \subseteq$ $\{1,2, \ldots, k\}$ of rows, we have a $\mathfrak{S}_{n}$-invariant

$$
\delta_{S}:=\prod_{i \in S} \theta_{i, 1}+\prod_{i \in S} \theta_{i, 2}+\cdots+\prod_{i \in S} \theta_{i, n} \in \wedge\left\{\Theta_{k \times n}\right\}
$$

where the products are taken in increasing order of $i \in S$. When $|S|$ is even, this has the potential to be a Lefschetz element, but $\delta_{S}^{2}=0$ when $|S|$ is odd. For $|S|=1$, the row sum $\delta_{S}$ may be easy to handle, but the situation becomes more complicated as an odd-sized set $S$ grows. Furthermore, one would have to understand how the various images of multiplication by the $\delta_{S}$ between bidegrees intersect as $S$ varies. 


\section{ACKNOWLEDGEMENTS}

B. Rhoades was partially supported by NSF Grant DMS-1500838. The authors thank Jim Haglund, Eugene Gorsky, Vic Reiner, Vasu Tewari, and Mike Zabrocki for helpful conversations and help with references. B. Rhoades is very grateful to Vic Reiner for a very helpful Zoom conversation about, among other things, Casimir elements.

\section{REFERENCES}

[1] K. Adiprasito, J. Huh, and E. Katz. Hodge theory for combinatorial geometries. Ann. of Math., 3 (2018), 3079-3098.

[2] D. Armstrong, V. Reiner, and B. Rhoades. Parking spaces. Adv. Math., 269 (2015), 647-706.

[3] F. Bergeron. $\left(G L_{k} \times S_{n}\right)$-Modules of Multivariate Diagonal Harmonics. Preprint, 2020. arXiv:2003.07402.

[4] M. Bremner. Free associative algebras, noncommutative Gröbner bases, and universal associative envelopes for nonassociative structures. arXiv:1303.0920.

[5] S. Billey, B. Rhoades, and V. Tewari. Boolean product polynomials, Schur positivity, and Chern plethysm. To appear, Int. Math. Res. Notices (IMRN), 2020. arXiv:1902.11165.

[6] E. Carlsson and A. Oblomkov. Affine Schubert calculus and double coinvariants. Preprint, 2018. arXiv:1801.09033.

[7] J. Haglund, J. Remmel, and A. T. Wilson. The Delta Conjecture. Trans. Amer. Math. Soc., 370, (2018), 40294057.

[8] J. Haglund, B. Rhoades, and M. Shimozono. Ordered set partitions, generalized coinvariant algebras, and the Delta Conjecture. Adv. Math., 329, (2018), 851-915.

[9] J. Haglund and E. Sergel. Schedules and the Delta Conjecture. Preprint, 2019. arXiv:1908.04732

[10] M. Haiman. Vanishing theorems and character formulas for the Hilbert scheme of points in the plane. Invent. Math., 149 (2), (2002), 371-407.

[11] M. Hara and J. Watanabe. The determinants of certain matrices arising from the Boolean lattice. Discrete Math., 308 (23), (2008), 5815-5822.

[12] R. Kane. Reflection Groups and Invariant Theory. CMS Books in Mathematics. Springer-Verlag, New York. 2001.

[13] T. Maeno, Y. Numata, and A. Wachi. Strong Lefschetz elements of the coinvariant rings of finite Coxeter groups. Algebr. Represent. Th., 14 (4), (2007), 625-638.

[14] B. Pawlowski and B. Rhoades. A flag variety for the Delta Conjecture. Trans. Amer. Math. Soc., 372, (2019), 8195-8248.

[15] B. Pawlowski, E. Ramos, and B. Rhoades. Spanning subspace configurations and representation stability. Preprint, 2019. arXiv:1907.07268

[16] B. Rhoades and A. T. Wilson. Vandermondes in Superspace. To appear, Trans. Amer. Math. Soc., 2020. arXiv: 1906.03315

[17] M. Rosas. The Kronecker product of Schur functions indexed by two-row shapes or hook shapes. J. Algebraic Combin., 14 (2), (2001), 153-173.

[18] J. Swanson and N. Wallach. Harmonic differential forms for pseudo-reflection groups I. Semi-invariants. Preprint, 2020. arXiv:2001.06076

[19] M. Zabrocki. A module for the Delta conjecture. Preprint, 2019. arXiv:1902.08966

[20] M. Zabrocki. Coinvariants and harmonics. Blog for Open Problems in Algebraic Combinatorics 2020. https://realopacblog.wordpress.com/2020/01/26/coinvariants-and-harmonics/.

Department of Mathematics

University of Pennsylvania

Philadelphia, PA, 19104-6395, USA

E-mail address: jk1093@math.upenn.edu

Department of Mathematics

University of California, San Diego

LA Jolla, CA, 92093-0112, USA

E-mail address: bprhoades@math.ucsd.edu 